\title{
NUMERICAL STUDY OF COMPRESSIBLE MAGNETOHYDRODYNAMIC TURBULENCE IN TWO DIMENSIONS
}

\author{
Hyesook Lee, ${ }^{1}$ Dongsu Ryu, ${ }^{1}$ Jongsoo Kim, ${ }^{2}$ T. W. Jones, ${ }^{3}$ and Dinshaw Balsara 4 \\ Received 2002 July 26; accepted 2003 May 9
}

\begin{abstract}
We have studied forced turbulence of compressible magnetohydrodynamic (MHD) flows through twodimensional simulations with different numerical resolutions. First, hydrodynamic turbulence with Mach number $\left\langle M_{s}\right\rangle_{\text {init }} \equiv\langle v\rangle_{\mathrm{rms}} / c_{s}=1$ and density compression $\langle\delta \rho / \rho\rangle_{\mathrm{rms}} \simeq 0.45$ was generated by enforcing a random force. Then, initial, uniform magnetic fields of various strengths were added with Alfvénic Mach number $\left\langle M_{\mathrm{A}}\right\rangle_{\text {init }} \equiv\langle v\rangle_{\mathrm{rms}} / c_{\mathrm{A} \text {,init }} \gg 1$. An isothermal equation of state was employed, and no explicit dissipation was included. In our simulations, the maximum amplification factor of magnetic energy depends on resolution and is proportional to $n_{x}^{1.32}$, where $n_{x}$ is the number of grid cells spanned by the computational box size. After the MHD turbulence is saturated, the resulting flows are categorized as very weak field (VWF), weak field (WF), and strong field (SF) classes, which have $\left\langle M_{\mathrm{A}}\right\rangle \equiv\langle v\rangle_{\mathrm{rms}} /\left\langle c_{\mathrm{A}}\right\rangle_{\mathrm{rms}} \gg 1,\left\langle M_{\mathrm{A}}\right\rangle>1$, and $\left\langle M_{\mathrm{A}}\right\rangle \sim 1$, respectively. The flow character in the VWF cases is similar to that of hydrodynamic turbulence. In the WF cases, the magnetic energy is still smaller than the kinetic energy in the global sense, but the magnetic field can become locally important. Hence, not only in the SF regime but also in the WF regime, turbulent transport is suppressed by the magnetic field. In the SF cases, the energy power spectra in the inertial range, although no longer power-law, exhibit a range with slopes close to $\sim 1.5$, hinting at the Iroshnikov-Kraichnan spectrum. These characteristics of the VWF, WF, and SF classes are consistent with their incompressible turbulence counterparts, indicating that a modest compressibility of $\langle\delta \rho / \rho\rangle_{\text {rms }} \lesssim 0.45$ or so does not play a significant role in turbulence. Our simulations were able to produce the SF-class behaviors only with a high resolution of at least $1024^{2}$ grid cells. With lower resolutions, we observed the formation of a dominant flux tube, which accompanies the separation of the magnetic field from the background flow. The specific requirements for the simulation of the SF class should depend on the code (and the numerical scheme) as well as the initial setup, but our results do indicate that very high resolution would be required for converged results in simulation studies of MHD turbulence.
\end{abstract}

Subject headings: methods: numerical — MHD — turbulence

\section{INTRODUCTION}

The existence of cosmic magnetic fields in diffuse astrophysical plasmas (the interstellar media in galaxies, the intracluster media in clusters of galaxies, and even the media associated with filaments and superclusters of galaxies) has been recognized for a while (for reviews, see, e.g., Kronberg 1994; Beck et al. 1996; Zweibel \& Heiles 1997; Carilli \& Taylor 2002; also see Ryu, Kang, \& Biermann 1998; Clarke, Kronberg, \& Böhringer 2001). Although the origin of such fields is not yet fully understood, turbulence is known to play an important role in the amplification and diffusion of existing magnetic fields. For instance, magnetic fields grow effectively by the turbulent motion of conducting fluids (the $\alpha$-effect), but they are not further amplified when Maxwell stresses become strong enough to affect the turbulent motion itself. How strong the magnetic fields would need to be in order to influence turbulence is one of the intriguing topics in magnetohydrodynamic (MHD) turbulence. It has

\footnotetext{
${ }^{1}$ Department of Astronomy and Space Science, Chungnam National University, Daejeon 305-764, Korea; 1hs@canopus.chungnam.ac.kr, ryu@canopus.chungnam.ac.kr.

${ }^{2}$ Korea Astronomy Observatory, 61-1, Hwaam-Dong, Yusong-Ku, Taejon 305-348, Korea; jskim@kao.re.kr.

${ }^{3}$ Department of Astronomy, University of Minnesota, Minneapolis, MN 55455; twj@msi.umn.edu.

${ }^{4}$ Department of Physics, University of Notre Dame, Notre Dame, IN 46556; dbalsara@nd.edu.
}

been argued through two-dimensional incompressible simulations that turbulent transport is reduced by weak magnetic fields whose energy is small compared to the kinetic energy of turbulent flows (Cattaneo \& Vainshtein 1991; Cattaneo 1994). A similar suppression was also observed in three-dimensional simulations of incompressible flows (Tao, Cattaneo, \& Vainshtein 1993). It is an important issue in astrophysics, since it is a part of the process for the generation and evolution of cosmic magnetic fields (see, e.g., Ruzmaikin, Shukurov, \& Sokolov 1988; Kulsrud et al. 1997; Kulsrud 1999).

In this work, we study compressible MHD turbulence by solving the ideal MHD equations with an isothermal equation of state. Since turbulent motion produces structures spanning a wide range of scales, with accompanying energy transfer among different scales, high spatial resolution is required to cover a sufficient inertial range. Hence, in this paper we present high-resolution two-dimensional simulations using up to $1536^{2}$ grid cells, leaving three-dimensional simulations for follow-up work. In addition, in order to achieve the highest possible magnetic Reynolds number and Reynolds number, no dissipation was included explicitly in our simulations. However, the resistivity and viscosity of numerical origin are still effective as small-scale dissipative channels. From the simulations, we examine the basic properties of the resulting MHD turbulence. We also address the dependence of the properties on resolution, including the amplification of the magnetic field. 
The rest of the paper is organized as follows: In $\S 2$ we describe the problem setup as well as the code properties. In $\S \S 3$ and 4 we present the results of simulations of hydrodynamic and MHD turbulence. Finally, in $\S 5$ the findings of this study are summarized.

\section{NUMERICS}

In simulations of compressible MHD turbulence, gas is heated by shocks and reconnection events, as well as numerical dissipation that mimics viscous and resistive influences (see below). Hence, in order to maintain the turbulence statistically in a steady state, cooling should be applied to the internal energy. One simple way to handle it is to assume the isothermality of flows. The MHD equations of compressible, isothermal gas are

$$
\begin{gathered}
\frac{\partial \rho}{\partial t}+\nabla \cdot(\rho \boldsymbol{v})=0 \\
\frac{\partial \boldsymbol{v}}{\partial t}+\boldsymbol{v} \cdot \boldsymbol{\nabla} \boldsymbol{v}+\frac{c_{s}^{2}}{\rho} \nabla \rho-\frac{1}{\rho}(\nabla \times \boldsymbol{B}) \times \boldsymbol{B}=\boldsymbol{f} \\
\frac{\partial \boldsymbol{B}}{\partial t}-\nabla \times(\boldsymbol{v} \times \boldsymbol{B})=0
\end{gathered}
$$

with an additional constraint,

$$
\nabla \cdot \boldsymbol{B}=0,
$$

for the absence of magnetic monopoles. Here, $c_{s}$ is the isothermal sound speed, and the units are chosen so that the factor of $4 \pi$ does not appear in the equations. To enforce turbulence, a random force per mass, $\boldsymbol{f}=f_{x} \hat{\boldsymbol{x}}+f_{y} \hat{\boldsymbol{y}}$, is added. It has the following form:

$$
\begin{aligned}
f_{x, y}(x, y, t)= & v_{\mathrm{amp}} \cos \left(\omega t+\delta_{t}\right) \cos \left(k_{\mathrm{in}, x} x+\delta_{x}\right) \\
& \times \cos \left(k_{\mathrm{in}, y} y+\delta_{y}\right),
\end{aligned}
$$

where $\delta_{t}, \delta_{x}$, and $\delta_{y}$ are random phases in the range of $0 \leq \delta_{t}, \delta_{x}, \delta_{y} \leq \pi$.

The above equations were solved using the multidimensional MHD code described in Kim et al. (1999), which is specifically designed for isothermal MHD. It is based on the explicit, finite-difference total variation diminishing scheme, which is a second-order accurate upwind scheme, and employs the minmod flux limiter. Simulations were performed in the computational domain of $x=[0, L]$, $y=[0, L]$, and $L=1$ with a periodic boundary condition using $n_{x}^{2}=256^{2}, 512^{2}, 1024^{2}$, and $1536^{2}$ grid cells. The total mass in the computational box is conserved, and the averaged density is set to be $\langle\rho\rangle=\rho_{0}=1$. The values of other parameters in the simulations are the following: the isothermal sound speed $c_{s}=1$, the angular frequency of the random forcing $\omega=2 \pi$, and the input wavenumbers $k_{\text {in }, x, y}=8 \pi$. With this choice, we note that the input scale of the random forcing is one-fourth of the computational box size, and the period is one sound-wave crossing time across the box. The amplitude of the random forcing, $v_{\mathrm{amp}}$, was set so that without a magnetic field, hydrodynamic turbulence is saturated with the averaged Mach number $\left\langle M_{s}\right\rangle_{\text {init }} \equiv\langle v\rangle_{\text {rms }} / c_{s}=1$ (see $\left.\S 3\right)$. MHD turbulence was generated by introducing uniform magnetic fields of various strength into the saturated hydrodynamic turbulence (see $\S 4)$.
Although our simulations do not contain explicit resistivity or viscosity, unavoidable numerical diffusion of the magnetic field and the momentum across the cell produce effective numerical resistivity and viscosity, respectively. Kim et al. (1999) studied the character of numerical dissipation in the code used for this work through the decay of a two-dimensional Alfvén wave. They showed that for waves spanning different numbers of grid cells $n$, the effective magnetic Reynolds number and the Reynolds number are proportional to $n^{1.66}$, mimicking the "hypertype" resistivity and viscosity $\left(\eta \nabla^{4} \boldsymbol{B}\right.$ and $\left.\mu \nabla^{4} \boldsymbol{v}\right)$ rather than the "normal-type" resistivity and viscosity $\left(\eta \nabla^{2} \boldsymbol{B}\right.$ and $\left.\mu \nabla^{2} \boldsymbol{v}\right)$ that characterize the physical dissipation of "collisional" fluids. They also estimated that the effective Reynolds numbers are larger than several hundreds if $n \geq 8$, so that the inertial range covers scales spanning eight grid cells or more. We note that numerical dissipation estimates depend somewhat on the tests used. For instance, the test suggested by Zweibel, Heitsch, \& Fan (2002) would have given somewhat smaller numerical dissipation. Hence, our estimation of the inertial range based on the Alfvén wave decay can be regarded to be rather conservative.

In most numerical studies of astrophysical turbulence based on explicit dissipation, the normal-type resistivity and viscosity have been included explicitly. However, dissipation processes in diffuse astrophysical plasmas are not well understood, and it is unlikely that the dissipation can be modeled by way of simple resistivity and viscosity coefficients. Hence, more careful consideration would be required in the studies of the properties of astrophysical turbulence when details on dissipative scales become important. In this paper we are concerned mostly with the properties of turbulence in the inertial range, which would be less sensitive to the form of dissipation, so we ignore dissipative complications outside this range.

\section{HYDRODYNAMIC TURBULENCE}

Simulations of hydrodynamic turbulence were first performed for two reasons. One was to generate the initial states for MHD simulations. The other was to observe the change of turbulence properties from the hydrodynamic case under the influence of magnetic fields. In the absence of magnetic fields, a transonic turbulence with $\left\langle M_{s}\right\rangle_{\text {init }} \simeq 1$ developed (see Table 1).

Figure 1 shows a typical resulting density distribution at the epoch $t=15$ and the time-averaged power spectrum of kinetic energy in the simulation with $1536^{2}$ grid cells. The existence of weak shocks is evident in the density image. Here, "weak" means the shock Mach number is small; typically $M_{\text {shock }} \sim 2$. The density compression is $\langle\delta \rho / \rho\rangle_{\text {rms }} \simeq 0.45$ when averaged over space and time in all the simulations of all resolutions (see Table 2). In a twodimensional system, the forced turbulence of "incompressible" flows exhibits the dual energy spectrum known as the Kraichnan spectrum: in the range of $k<k_{\text {in }}$, the slope follows $k^{-5 / 3}$, indicating the direct cascade of energy, while in the range of $k>k_{\text {in }}$, it follows $k^{-3}$, indicating the direct cascade of enstrophy (see, e.g., Lesieur 1997 and references therein). In the case of "high compressibility," in which strong shocks are common, the energy spectrum follows the $k^{-2}$ slope in the inertial range, known as the Burgers spectrum (see, e.g., Lesieur 1997 and references therein). In our results with weak compressibility, the energy spectrum is 
TABLE 1

Alfvén Mach Number $\left\langle M_{\mathrm{A}}\right\rangle$ and Mach Number $\left\langle M_{s}\right\rangle$ For SF, WF, and VWF Cases

\begin{tabular}{|c|c|c|c|c|c|c|c|c|c|c|c|c|}
\hline \multirow[b]{3}{*}{ Grid Cell Number } & \multicolumn{12}{|c|}{$\left\langle M_{\mathrm{A}}\right\rangle_{\text {init }}$} \\
\hline & \multicolumn{2}{|c|}{50} & \multicolumn{2}{|c|}{100} & \multicolumn{2}{|c|}{200} & \multicolumn{2}{|c|}{300} & \multicolumn{2}{|c|}{1000} & \multicolumn{2}{|c|}{$\infty^{\mathrm{a}}$} \\
\hline & $\left\langle M_{\mathrm{A}}\right\rangle$ & $\left\langle M_{s}\right\rangle$ & $\left\langle M_{\mathrm{A}}\right\rangle$ & $\left\langle M_{s}\right\rangle$ & $\left\langle M_{\mathrm{A}}\right\rangle$ & $\left\langle M_{s}\right\rangle$ & $\left\langle M_{\mathrm{A}}\right\rangle$ & $\left\langle M_{s}\right\rangle$ & $\left\langle M_{\mathrm{A}}\right\rangle$ & $\left\langle M_{s}\right\rangle$ & $\left\langle M_{\mathrm{A}}\right\rangle$ & $\left\langle M_{s}\right\rangle$ \\
\hline . & $2.38 \dagger$ & $0.83 \dagger$ & 4.93 & 0.95 & 9.70 & 0.99 & 14.90 & 1.00 & 49.66 & 1.00 & $\infty$ & 1.00 \\
\hline 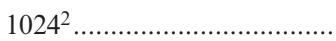 & 1.60 & 0.70 & $3.02 \dagger$ & $0.86 \dagger$ & 6.00 & 0.95 & 9.05 & 0.98 & 31.38 & 1.00 & $\infty$ & 1.00 \\
\hline $1536^{2}$ & 1.41 & 0.66 & $2.28 \dagger$ & $0.81 \dagger$ & $4.04 \dagger$ & $0.85 \dagger$ & 6.57 & 0.97 & $\ldots$ & $\ldots$ & $\infty$ & 1.00 \\
\hline
\end{tabular}

Notes.-Averaged spatially over the whole computational domain and temporally over $20 \leq t \leq t_{\text {end }}$ for $512^{2}$ and $1024^{2}$ simulations and over $10 \leq t \leq t_{\text {end }}$ for $1536^{2}$ simulations. The values for the WF cases are marked with a dagger for clarity. The simulations with $256^{2}$ grid cells were performed but are not listed.

a Hydrodynamic turbulence.

consistent with the Kraichnan spectrum, as shown in the right panel of Figure 1, but the slope for $k>k_{\text {in }}$ is $\sim 2.72$, somewhat shallower than for incompressible turbulence. As listed in Table 3, the deviation of the slope from $k^{-3}$ increases systematically as the resolution increases. This is because in higher resolution simulations, shocks are better resolved, and hence, their effect is more evident in the energy power spectrum.

\section{MHD TURBULENCE}

Weak, uniform magnetic fields of various strength were added to the fully developed hydrodynamic turbulence. In the $n_{x}^{2}=256^{2}, 512^{2}$, and $1024^{2}$ simulations, the flows of hydrodynamic turbulence at $t=30$ were taken as the initial states, while in the $1536^{3}$ simulations, the flow at $t=15$ (Fig. 1, right) was taken. The strength of the added magnetic fields was set so that the initial Alfvénic Mach number, $\left\langle M_{\mathrm{A}}\right\rangle_{\text {init }} \equiv\langle v\rangle_{\mathrm{rms}} / c_{\mathrm{A} \text {,init }}$, ranged from 1000 to 10 (corresponding to a plasma $\beta$ of $2 \times 10^{6}$ to 200 ). The Alfvénic Mach number represents the ratio of Reynolds stresses to
Maxwell stresses and so is a convenient way to characterize magnetic field strength in these flows.

The magnetic field strength grew rapidly, almost exponentially, during the initial transient period and then saturated. Figure 2 shows the time evolution of the rms magnetic field strength and flow velocity. The resulting flows of MHD turbulence after saturation can be naturally categorized into very weak field (VWF), weak field (WF), and strong field (SF) classes (Table 4). In the VWF cases, magnetic field influences are negligible, and the flow velocity does not change noticeably from purely hydrodynamic turbulence. In the WF cases, the magnetic field energy $E_{\mathrm{mag}}$ is still small compared to the flow kinetic energy $E_{\text {kin }}$, but the magnetic field does locally affect small-scale flow motions. Hence, the turbulence flow velocity is decreased from that of nonmagnetic cases. Finally, the cases in which $E_{\mathrm{mag}}$ becomes comparable to $E_{\text {kin }}$ are classified as the SF class. We note that the change in the flow properties is gradual with the increase of the strength of the initial, uniform fields. Hence, boundaries between the classes are somewhat arbitrary. We set the criterion for the boundary between the VWF and WF classes as
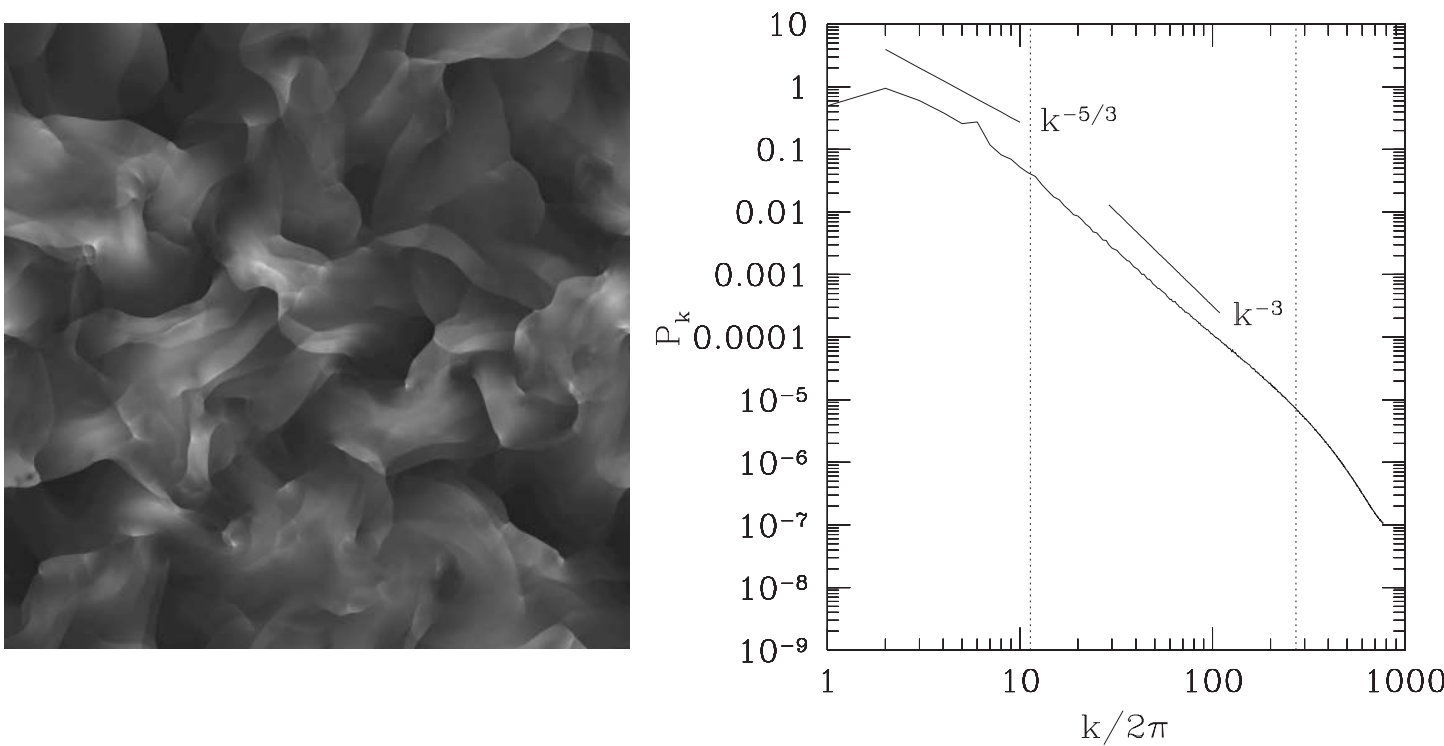

Fig. 1. - Left: Gray-scale image of density for the nonmagnetic case with $1536^{2}$ resolution. Brighter regions correspond to higher values, and the gray scale was set arbitrarily to highlight structures. The image represents the density distribution typical at the epoch $(t=15)$ after hydrodynamic turbulence is fully developed. Right: Power spectrum of the kinetic energy, time-averaged over $10 \leq t \leq t_{\text {end }}$, for the nonmagnetic case with $1536^{2}$ resolution. Here, $k \equiv\left(k_{x}^{2}+k_{y}^{2}\right)^{1 / 2}$. The peak at $k=5.7(2 \pi)$ corresponds to the wavenumber of the power input scale, $k_{\text {in }} \equiv\left(k_{\text {in } x}^{2}+k_{\text {in } y}^{2}\right)^{1 / 2}$. For reference, two vertical lines are drawn at $k=2 k_{\text {in }}$ and the wavenumber corresponding to eight grid cells, $k_{8}$. Two lines with slopes of $-5 / 3$ and -3 are drawn for comparison. 
TABLE 2

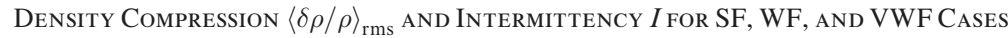

\begin{tabular}{|c|c|c|c|c|c|c|c|c|c|c|c|c|}
\hline \multirow[b]{2}{*}{ GRID CELl Number } & \multicolumn{12}{|c|}{$\left\langle M_{\mathrm{A}}\right\rangle_{\text {init }}$} \\
\hline & $\langle\delta \rho / \rho\rangle_{\mathrm{rms}}$ & $I$ & $\langle\delta \rho / \rho\rangle_{\mathrm{rms}}$ & $I$ & $\langle\delta \rho / \rho\rangle_{\mathrm{rms}}$ & $I$ & $\langle\delta \rho / \rho\rangle_{\mathrm{rms}}$ & $I$ & $\langle\delta \rho / \rho\rangle_{\mathrm{rms}}$ & $I$ & $\langle\delta \rho / \rho\rangle_{\mathrm{rms}}$ & $I$ \\
\hline $512^{2}$. & $0.32 \dagger$ & $2.68 \dagger$ & 0.39 & 3.86 & 0.42 & 4.80 & 0.42 & 5.28 & 0.45 & 5.93 & 0.45 & \\
\hline $1024^{2}$. & 0.25 & 2.06 & $0.34 \dagger$ & $3.03 \dagger$ & 0.40 & 4.28 & 0.42 & 5.05 & 0.44 & 5.88 & 0.45 & \\
\hline . & 0.24 & 1.95 & $0.30 \dagger$ & $2.48 \dagger$ & $0.37 \dagger$ & $3.48 \dagger$ & 0.41 & 4.58 & $\ldots$ & $\ldots$ & 0.45 & \\
\hline
\end{tabular}

NOTES.-Averaged spatially over the whole computational domain and temporally over $20 \leq t \leq t_{\text {end }}$ for $512^{2}$ and $1024^{2}$ simulations and over $10 \leq t \leq t_{\text {end }}$ for $1536^{2}$ simulations. The values for the WF cases are marked with a dagger for clarity. The simulations with $256^{2}$ grid cells were performed but are not listed.

a Hydrodynamic turbulence.

$\left\langle M_{s}\right\rangle \equiv\langle v\rangle_{\mathrm{rms}} / c_{s} \lesssim 1 ;$ more specifically, $\left\langle M_{s}\right\rangle=0.9$ was taken. On the other hand, the criterion for the boundary between the WF and SF classes was set as $\left\langle M_{\mathrm{A}}\right\rangle \equiv\langle v\rangle_{\mathrm{rms}} /\left\langle c_{\mathrm{A}}\right\rangle_{\mathrm{rms}} \gtrsim 1$; more specifically, $\left\langle M_{\mathrm{A}}\right\rangle=2$ was taken. Among the SF cases, we observed examples in which a change in the magnetic field configuration takes place. In particular, there are cases in which a dominant flux tube develops. These cases we categorized specifically as the field separation (FS) class. The growth of magnetic field strength and the characteristics of the four classes are further detailed in the following subsections.

For quantitative discussions, we computed, in addition to $\left\langle M_{\mathrm{A}}\right\rangle$ and $\left\langle M_{s}\right\rangle$ (Table 1$)$, the density compression $\langle\delta \rho / \rho\rangle_{\text {rms }}$ and the intermittency (or the kurtosis of the field distribution) $I \equiv\left\langle B^{4}\right\rangle_{\text {rms }} /\left\langle B^{2}\right\rangle_{\text {rms }}^{2}$ (Table 2), as well as the slopes of the power spectra of the kinetic and total energies (Table 3). Note that with a Gaussian distribution of the magnetic field, $\boldsymbol{B} \propto \exp \left(-x^{2} / 2 \sigma^{2}\right) \hat{\boldsymbol{y}}$, the value of $I$ is 2.39 when summed over the interval $-3 \sigma \leq x \leq 3 \sigma$, or 3.99 when $-5 \sigma \leq x \leq 5 \sigma$ is taken into account.

\subsection{Growth of the Magnetic Field Strength}

There is no dynamo action in two dimensions, and the total magnetic flux through given boundaries is conserved. Hence, the growth of the magnetic field strength shown in Figure 2 is due to stretching and compression. The growth stops either when the magnetic energy reaches an equipartition with the kinetic energy, so that the back-reaction from magnetic field Maxwell stresses plays a dominant role, or when the separation between magnetic sheets is reduced to the diffusive scale, so that reconnection takes place (see, e.g.,
Biskamp 1993 for details). If the initial, uniform magnetic field is weak enough, the growth is saturated by the latter cause before the magnetic energy reaches equipartition, as in the VWF and WF cases. Then, the resulting growth should depend on the diffusive scale and, hence, on the effective resistivity. Then, the amplification of magnetic energy from the initial value is expected to be $\lesssim\left(k_{\text {in }} \delta\right)^{-2} \sim \operatorname{Re}_{m}$ (see, e.g., Biskamp 1993). Here, $\delta$ is the diffusive scale, and $\mathrm{Re}_{m}$ is the magnetic Reynolds number.

Figure 3 shows the averaged amplification of magnetic energy in our simulations as a function of grid resolution. Only the VWF and WF cases were considered, because in the SF cases, the growth of the magnetic field stopped as a consequence of Maxwell stresses, rather than reconnection. The amplification shown in the figure is proportional to $n_{x}^{1.32}$. Furthermore, with $\operatorname{Re}_{m} \propto n^{1.66}$ for our code (see $\S 2$ ), the amplification is estimated to be approximately proportional to $\mathrm{Re}_{m}^{0.8}$, which is somewhat shallower than the expected dependence $\left(\propto \mathrm{Re}_{m}\right)$. However, this result agrees well with results of two-dimensional numerical simulations for incompressible MHD turbulence (Biskamp 1993).

\subsection{VWF Cases}

The VWF class includes those cases in which the backreaction of the magnetic field into flow motions is insignificant. The criterion $\left\langle M_{s}\right\rangle \gtrsim 0.9$ corresponds to an rms velocity decrease by less than $10 \%$. The characteristics of turbulence in these cases are summarized as follows: (1) The density compression is still large, with $\langle\delta \rho / \rho\rangle_{\text {rms }} \gtrsim 0.38$ (Table 2), and shocks still exist, as shown clearly in the bottom left panel of Figure 4. (2) The intermittency is large,

TABLE 3

Spectral Slope of Kinetic Energy and Total Energy for SF, WF, and VWF Cases

\begin{tabular}{|c|c|c|c|c|c|c|c|c|c|c|c|c|}
\hline \multirow{2}{*}{ GRID Cell Number } & \multicolumn{12}{|c|}{$\left\langle M_{\mathrm{A}}\right\rangle_{\text {init }}$} \\
\hline & \multicolumn{2}{|c|}{50} & \multicolumn{2}{|c|}{100} & \multicolumn{2}{|c|}{200} & \multicolumn{2}{|c|}{300} & \multicolumn{2}{|c|}{1000} & \multicolumn{2}{|c|}{$\infty^{\mathrm{a}}$} \\
\hline $512^{2}$. & $2.22 \dagger$ & $1.27 \dagger$ & 2.77 & 2.21 & 2.95 & 2.78 & 2.67 & 2.61 & 2.77 & 2.76 & 2.90 & 2.90 \\
\hline $1024^{2}$ & 1.46 & 1.27 & $2.07 \dagger$ & $1.20 \dagger$ & 2.74 & 2.09 & 2.84 & 2.51 & 2.75 & 2.71 & 2.85 & 2.85 \\
\hline
\end{tabular}

Notes.-Averaged temporally over $20 \leq t \leq t_{\text {end }}$ for $512^{2}$ and $1024^{2}$ simulations and over $10 \leq t \leq t_{\text {end }}$ for $1536^{2}$ simulations. Slopes were fitted over the range of $2 k_{\text {in }} \leq k \leq k_{24}$. Here, $k_{\text {in }}$ and $k_{24}$ are the wavenumbers corresponding to the power input scale and 24 grid cells, respectively. The values for the WF cases are marked with a dagger for clarity. The simulations with $256^{2}$ grid cells were performed but are not listed.

a Hydrodynamic turbulence. 

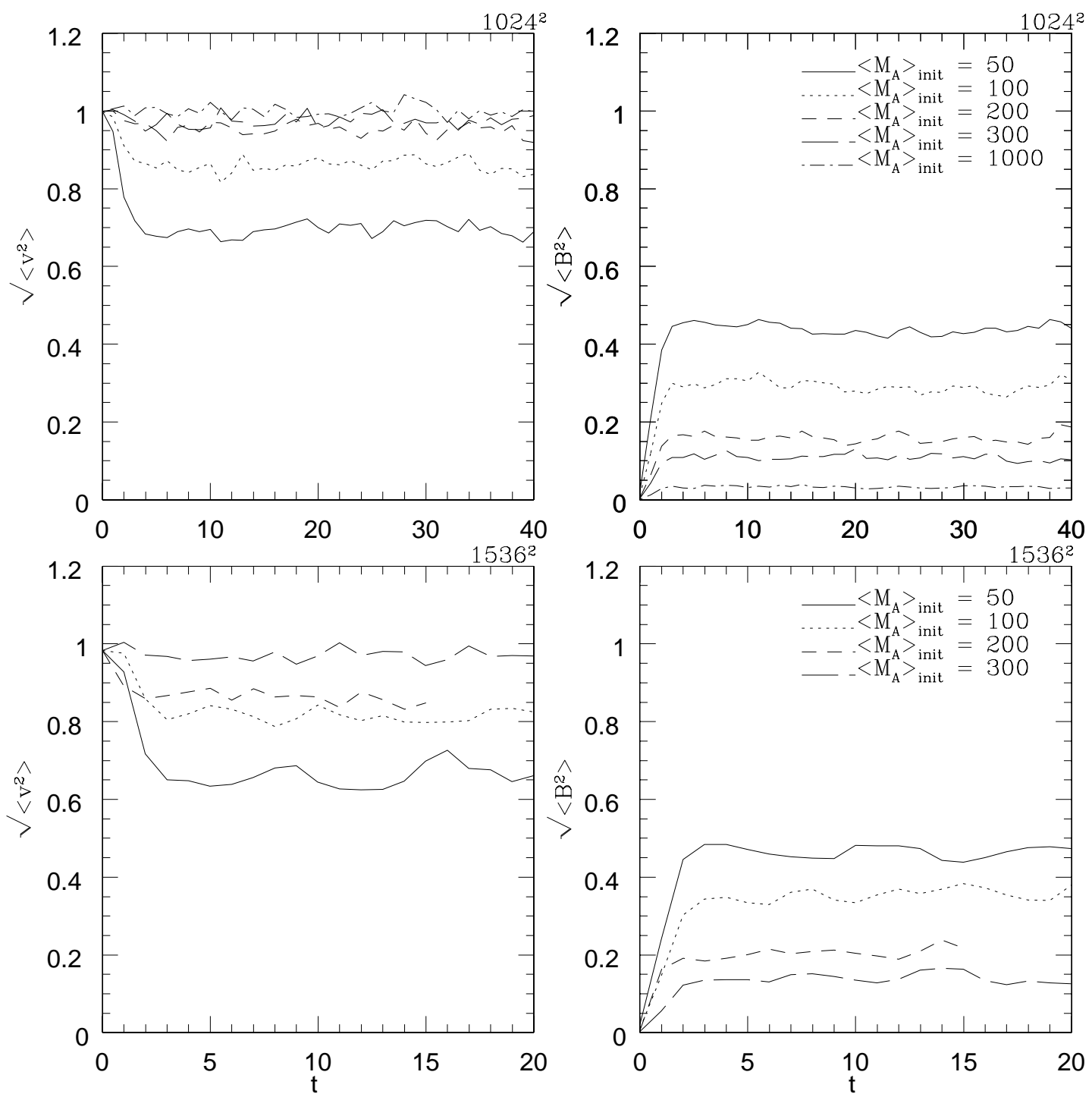

FIG. 2.-Time evolution of rms velocity $(l e f t)$ and magnetic field strength $(r i g h t)$, which were averaged over 1 period of random forcing $(\tau=1)$, for different initial Alfvénic Mach numbers. Top: Cases with $1024^{2}$ resolution. Bottom: Cases with $1536^{2}$ resolution.

with $I \gtrsim 3.5$ (Table 2), which indicates that the spatial contrast in the magnetic field strength distribution is high. In particular, the bottom right panel of Figure 4 shows that the magnetic field is mostly thin tubes (or thin sheets in extension to the third dimension). (3) The Alfvén Mach number is $\left\langle M_{\mathrm{A}}\right\rangle \lesssim 4.5$ (Table 1), and hence the kinetic energy is at least an order of magnitude larger than the magnetic energy. In addition, the power of the kinetic energy, $P_{k}^{\text {kin }}$, is larger than that of the magnetic energy, $P_{k}^{\text {mag }}$, over most wavenumbers, as shown in the bottom panels of Figure 5. However, $P_{k}^{\mathrm{mag}} \gtrsim P_{k}^{\mathrm{kin}}$ in a small range of large wavenumbers. This is because the magnetic field first built up on small

TABLE 4

Classification of Turbulence/Time at the End of Simulations, $t_{\text {end }}$

\begin{tabular}{|c|c|c|c|c|c|c|c|c|c|c|c|c|c|c|c|c|}
\hline $\begin{array}{c}\text { GRID CELL } \\
\text { Number }\end{array}$ & \multicolumn{2}{|c|}{10} & \multicolumn{2}{|c|}{20} & \multicolumn{2}{|c|}{50} & \multicolumn{2}{|c|}{100} & \multicolumn{2}{|c|}{200} & \multicolumn{2}{|c|}{300} & \multicolumn{2}{|c|}{1000} & \multicolumn{2}{|c|}{$\infty$} \\
\hline $512^{2}$. & FS & 80 & FS & 100 & $\mathrm{WF} \dagger$ & $40 \dagger$ & VWF & 40 & VWF & 40 & VWF & 40 & VWF & 40 & HD & 40 \\
\hline $1024^{2}$. & FS & 110 & FS & 140 & SF & 40 & $\mathrm{WF} \dagger$ & $40 \dagger$ & VWF & 40 & VWF & 40 & VWF & 40 & HD & 40 \\
\hline
\end{tabular}

NoтES.-The turbulence classifications are field separation, strong field, weak field, very weak field, and hydrodynamic. See the text for details. The values for the WF cases are marked with a dagger for clarity. The simulations with $256^{2}$ grid cells were performed but are not listed. 


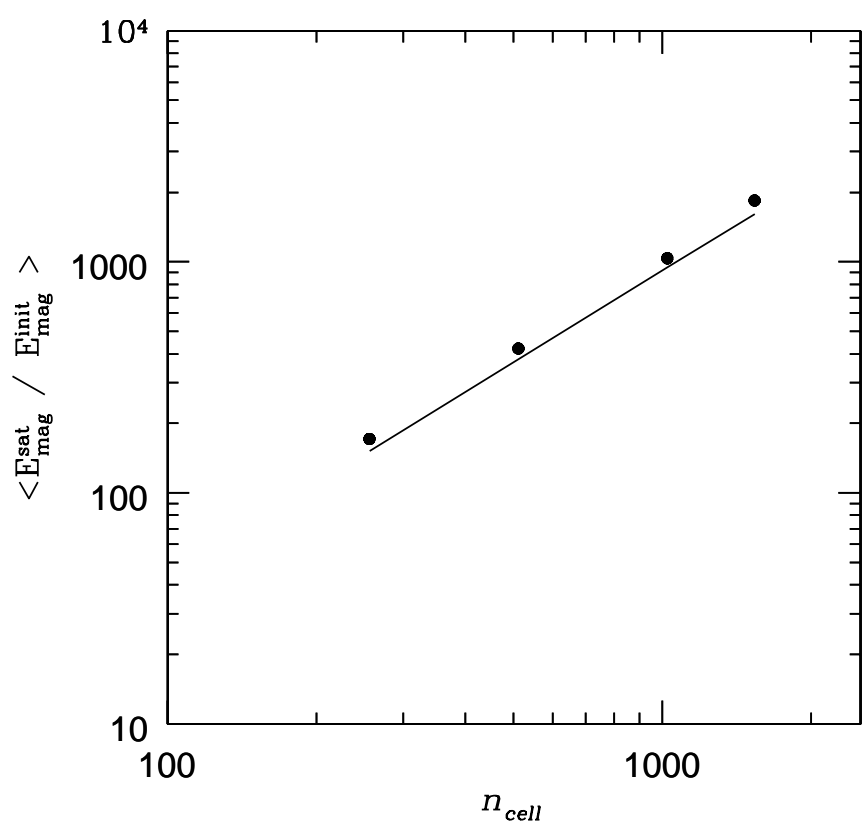

FIG. 3.-Averaged amplification of the magnetic energy from the initial value in the VWF and WF cases as a function of numerical resolution. Four points of $256^{2}, 512^{2}, 1024^{2}$, and $1536^{2}$ grid cells are plotted. The fitted line $\propto n_{x}^{1.32}$ is drawn.

scales. However, it saturated because of reconnection before the power could extend to larger scales. (4) The slope of $P_{k}^{\text {kin }}$ was not affected much by the magnetic field. The change was less than a few percent, as shown in Table 3. However, the slope of the power of the total energy, $P_{k}^{\text {tot }}$, changed significantly compared to the hydrodynamic case, especially in the MHD cases close to the boundary between the VWF and WF classes. This is because the small-scale power of the magnetic energy pushed $P_{k}^{\text {tot }}$ up, and hence $P_{k}^{\text {tot }}$ does not have a single, well-defined slope any more.

\subsection{WF Cases}

The WF class was categorized by the property that globally the magnetic energy is smaller than the kinetic energy, but yet the back-reaction of the magnetic field is not negligible. As the criteria to represent this property, $\left\langle M_{s}\right\rangle \lesssim 0.9$ and $\left\langle M_{\mathrm{A}}\right\rangle \gtrsim 2$ were adopted (Table 1). The characteristics of turbulence in these cases are the following: (1) As shown in the middle left panel of Figure 4, the occurrence of shocks is reduced and their strength is weakened compared to the hydrodynamic case. The density compression is $0.28 \lesssim$ $\langle\delta \rho / \rho\rangle_{\text {rms }} \lesssim 0.38$ (Table 2), which is smaller than in the VWF cases. (2) The contours of magnetic field lines plotted in the middle right panel of Figure 4 show the appearance of almost circular magnetic islands. At the same time, the magnetic field is less concentrated than in the VWF cases, with smaller intermittency $2.2 \lesssim I \lesssim 3.5$ (Table 2). (3) Although in the small-wavenumber region the magnetic energy power is still smaller than the kinetic energy power, $P_{k}^{\text {mag }} \ll P_{k}^{\text {kin }}$, at large wavenumbers, $P_{k}^{\mathrm{mag}} \gtrsim P_{k}^{\mathrm{kin}}$, as shown in the middle panels of Figure 5. Hence, turbulent transport can be suppressed by magnetic fields on small scales where $P_{k}^{\text {mag }} \gtrsim P_{k}^{\text {kin }}$. Consequently, the flow velocity decreases noticeably from that of hydrodynamic turbulence (see Fig. 2). (4) The power of the magnetic energy, $P_{k}^{\mathrm{mag}}$, changed the shape of $P_{k}^{\text {tot }}$ as well as of $P_{k}^{\text {kin }}$ in power spectra, and the slopes are not simply defined over the entire inertial range of wavenumbers any more (see Fig. 5). We calculated the slopes of $P_{k}^{\mathrm{kin}}$ and $P_{k}^{\text {tot }}$ over $2 k_{\text {in }} \leq k \leq k_{24}$, where the slopes are relatively well defined, and listed them in Table 3. Here, $k_{24}$ is the wavenumber corresponding to 24 grid cells. The slope of $P_{k}^{\mathrm{kin}}$ is significantly smaller than those of the hydrodynamic and VWF cases. The slope of $P_{k}^{\text {tot }}$ is quite small, but this is because $P_{k}^{\mathrm{mag}}$ peaks in the wavenumber range over which the slope was calculated (see Fig. 5).

\subsection{SF Cases}

In the SF cases, the magnetic energy grows to become comparable to the kinetic energy, with $\left\langle M_{\mathrm{A}}\right\rangle \lesssim 2$ at saturation (Table 1). Hence, the flow velocity is influenced significantly by the magnetic field (Fig. 2), with $\left\langle M_{s}\right\rangle \lesssim 0.75$ at saturation (Table 1). The characteristics of SF turbulence are the following: (1) With the significantly reduced Mach number, shocks are rare, as shown in the top left panel of Figure 4 . The density compression is accordingly reduced to $\langle\delta \rho / \rho\rangle_{\text {rms }} \lesssim 0.28$ (Table 2). (2) As shown in the top right panel of Figure 4, circular magnetic flux islands, or loops, are common. This trend of changing topology in magnetic field lines, from tubes to loops, with an increase of magnetic field strength was observed also in the two-dimensional simulations of incompressible MHD turbulence (see, e.g., Biskamp 1993). At the same time, the spatial contrast of the magnetic field strength distribution becomes low with smaller intermittency $I \lesssim 2.2$ (Table 2 ). (3) The power of the magnetic energy exceeds that of the kinetic energy with $P_{k}^{\text {mag }} \gtrsim P_{k}^{\text {kin }}$ over all $k \gtrsim k_{\text {in }}$, as shown in the top panels of Figure 5, although still $P_{k}^{\text {mag }}<P_{k}^{\mathrm{kin}}$ for $k \lesssim k_{\text {in }}$. (4) The slopes of $P_{k}^{\mathrm{kin}}$ and $P_{k}^{\text {tot }}$, calculated over $2 k_{\text {in }} \leq k \leq k_{24}$ as in the WF cases, are listed in Table 3 . Across the above wavenumber range, $P_{k}^{\mathrm{kin}}, P_{k}^{\mathrm{tot}}$, and $P_{k}^{\mathrm{mag}}$ all exhibit a single slope close to $\sim 1.5$, namely, the slope of the IroshnikovKraichnan spectrum (Fig. 5, top).

We note that the emergence of the slope of $\sim 1.5$ is achieved only in the very high resolution simulations with $1024^{2}$ and $1536^{2}$ grid cells, not in the simulations with $256^{2}$ and $512^{2}$ grid cells. In fact, there is no case categorized as the SF class in the simulations with $256^{2}$ and $512^{2}$ grid cells at all (see Table 4). That is, our two-dimensional simulations of MHD turbulence start to show converged behaviors with $1024^{2}$ grid cells or more.

\subsection{FS Cases}

The cases with $\left\langle M_{\mathrm{A}}\right\rangle_{\text {init }} \lesssim 30$ show FS-class behavior, wherein a dominant flux tube forms and, as a consequence, the magnetic field separates from the background flow (see Fig. 6). In such FS cases, flows become anisotropic, and their characteristics are different from the other cases. The appearance of this behavior can be understood as follows: Recall that there are three ideal two-dimensional MHD invariants: the total energy $E_{\mathrm{tot}}$, the mean square of the magnetic potential $A$, and the cross-helicity

$$
K=\frac{1}{2} \int \boldsymbol{v} \cdot \boldsymbol{B} d^{2} \boldsymbol{x}
$$

Among them, the power spectrum of $A$ exhibits an inverse cascade, while the power spectra of $E_{\text {tot }}$ and $K$ exhibit a normal cascade (see, e.g., Biskamp 1993). Hence, just as in three dimensions, large-scale magnetic field power can be built up in the regime $k \leq k_{\text {in }}$ in two-dimensional MHD turbulence. 

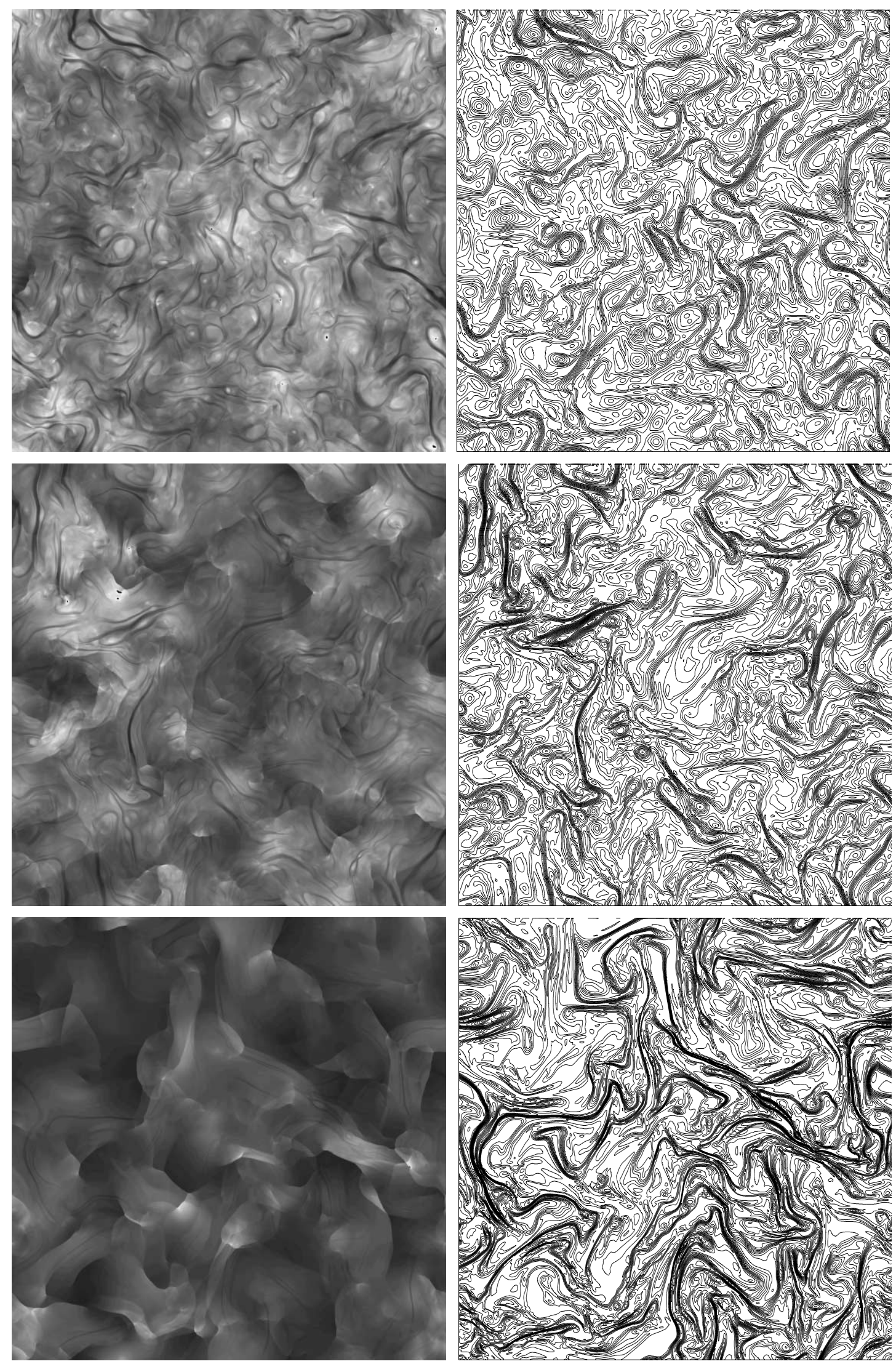

FIG. 4. - Gray-scale images of density (left $)$ and contours of magnetic field lines (right) at the epoch $t=13$ for cases with $1536^{2}$ resolution. Top: Case of $\left\langle M_{\mathrm{A}}\right\rangle_{\text {init }}=50$, classified as the SF class. Middle: Case of $\left\langle M_{\mathrm{A}}\right\rangle_{\text {init }}=100$, classified as the WF class. Bottom: Case of $\left\langle M_{\mathrm{A}}\right\rangle_{\text {init }}=300$, classified as the VWF class. In the density images, brighter regions represent higher values, and the gray scale was set arbitrarily to highlight structures. 

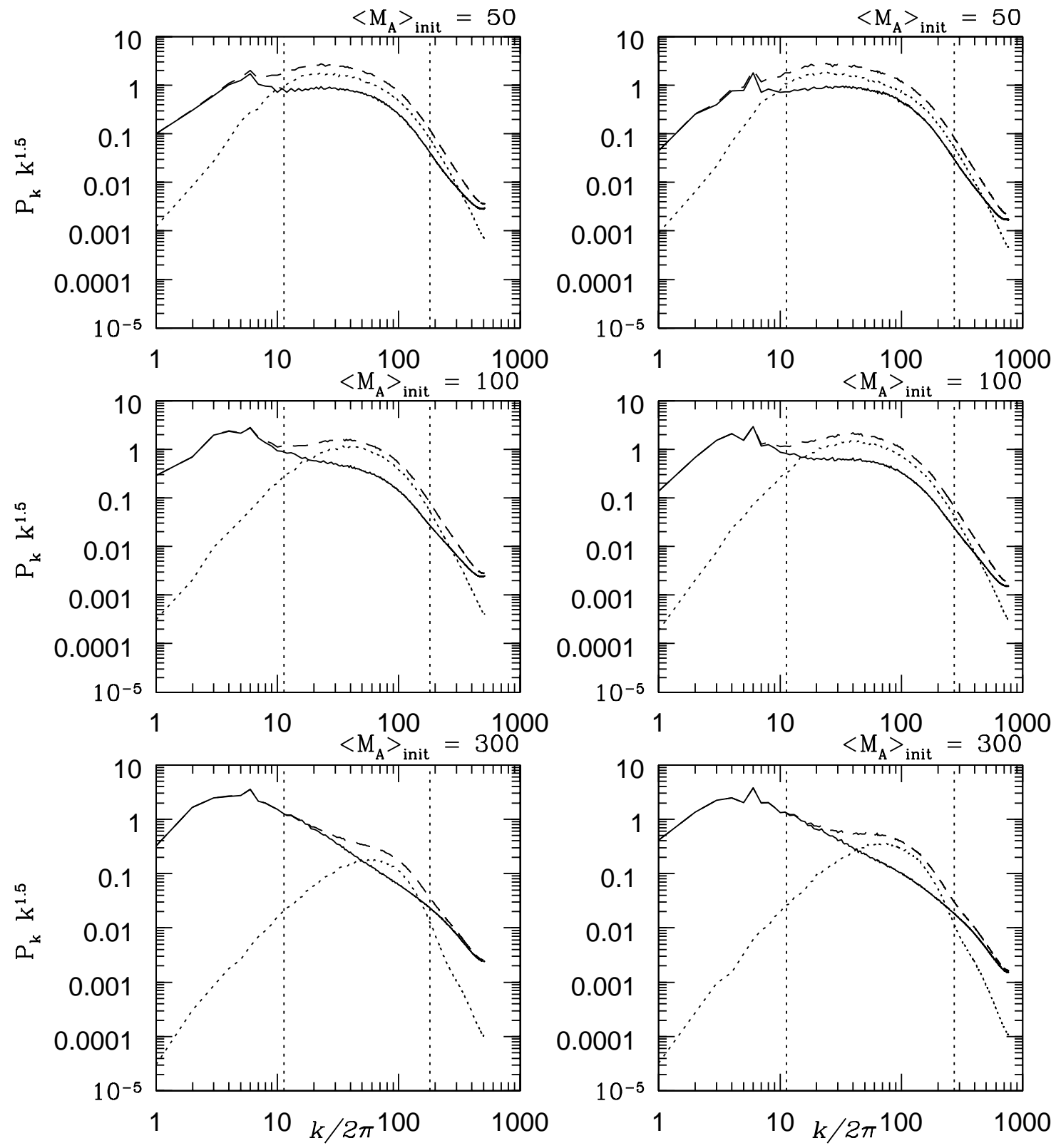

FIG. 5.-Power spectra, time-averaged over $20 \leq t \leq t_{\text {end }}$ for $1024^{2}$ resolution (left) and over $10 \leq t \leq t_{\text {end }}$ for $1536^{2}$ resolution (right). Spectra are

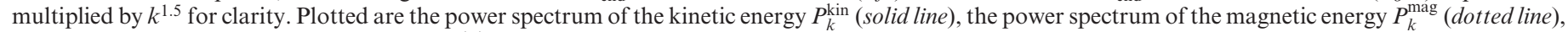

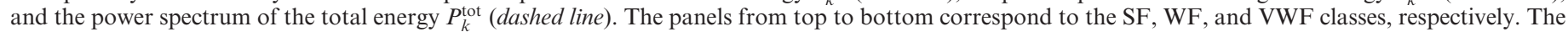
slopes fitted over the range of $2 k_{\text {in }} \leq k \leq k_{24}$ are shown in Table 3 . Here $k_{24}$ is the wavenumber corresponding to 24 grid cells.

In our FS cases this power buildup proceeds through the formation of a magnetic flux tube with a coherent length larger than the scale associated with $k_{\text {in }}$. However, as pointed out in $\S 4.1$, there is no dynamo action in our simulations. Hence, the formation of such a flux tube should involve reconnection followed by the expulsion of gas out of the flux tube, reducing the gas density (therefore, also the gas pressure) inside the tube, while maintaining approximate pressure equilibrium.

We note that the critical value of $\left\langle M_{\mathrm{A}}\right\rangle_{\text {init }}$ for the FS class is independent of numerical resolution in our simulations (see Table 4). This is because the existence of the flux tube depends on its ability to resist ram pressure bending, which depends on large-scale flows, and so is independent of numerical resolution. On the other hand, since the formation of the dominant flux tube involves reconnection, we expect the time to reach the state in which the magnetic field separates from the background flow to depend on resistivity. The flux tube was developed in a few tens of eddy turnover times in our simulations. However, in astrophysical environments such as interstellar media or intracluster media, the resistivity is much smaller than that of our simulations. The classical magnetic Reynolds number in the interstellar medium can be as large as $10^{50}$ or so (see, e.g., Spitzer 1979). Hence, we expect that the emergence of the flux tube would take too long in astrophysical environments, and instead, turbulence of the SF class is more likely to develop. Therefore, the failure of SF cases in our $256^{2}$ 

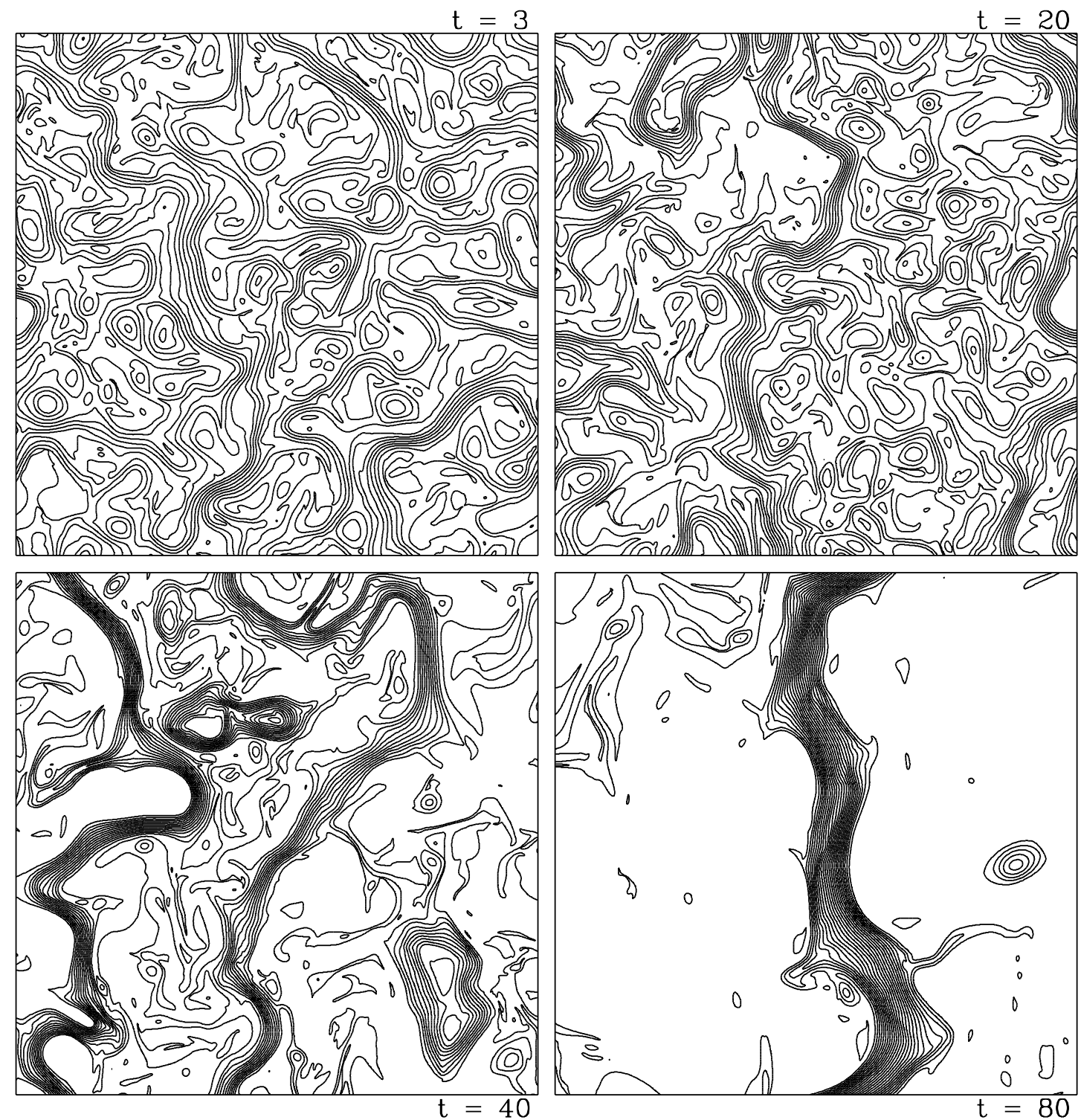

FIG. 6. - Contours of magnetic field lines at four different times for the case of $\left\langle M_{\mathrm{A}}\right\rangle_{\text {init }}=10$ with $1024^{2}$ resolution. It belongs to the FS class.

and $512^{2}$ simulations is probably a limitation of numerical simulations: in particular, from very large numerical resistivity compared to astrophysical environments and perhaps also from the periodic boundary we employed.

\section{SUMMARY AND CONCLUSION}

We performed high-resolution two-dimensional simulations of isothermal MHD turbulence using up to $1536^{2}$ grid cells. Compressibility was taken into account, with Mach numbers $\left\langle M_{s}\right\rangle \lesssim 1$. In order to maximize the magnetic Reynolds number and the Reynolds number, no explicit dissipation was included. Instead, resistivity and viscosity of numerical origin were utilized. Our findings are summarized as follows:

1. The growth of magnetic energy from the initial value depends on the effective resistivity and, hence, on resolution. We measured a maximum magnetic energy amplification proportional to $n_{x}^{1.32}$. After the growth saturated, the turbulence could be categorized into three classes, which we labeled very weak field (VWF), weak field (WF), and strong field (SF) classes, depending on the strength of the uniform component of the magnetic fields, or equivalently, the initial Alfvén Mach number $\left\langle M_{\mathrm{A}}\right\rangle_{\text {init }}$. Each class is characterized by different turbulence properties.

2. In the WF cases, although $E_{\mathrm{mag}}<E_{\mathrm{kin}}$ globally, the rms flow velocity decreases because of the magnetic field, which can become locally important. That is, even a weak magnetic field reduces turbulent transport (Cattaneo \& Vainshtein 1991; Cattaneo 1994).

3. Although the inertial range power spectra of MHD turbulence are not represented by power laws any more, part of the inertial range still develops a slope close to $\sim 1.5$ : that of the Iroshnikov-Kraichnan spectrum, in the SF cases. Such a slope develops only in the highest resolution simulations with $1024^{2}$ and $1536^{2}$ grid cells.

4. In the lower resolution simulations with $256^{2}$ and $512^{2}$ grid cells, there are no cases categorized as the SF class. Instead, with $\left\langle M_{\mathrm{A}}\right\rangle_{\text {init }} \lesssim 30$, those simulations developed into FS cases, in which a large flux tube dominates the flow 
structures and separates the flow from the magnetic field. This FS property is the result of large numerical resistivity along with the periodic box used.

The conclusions of our work are the following:

1. For the VWF, WF, and SF cases, we observed properties of turbulence that are consistent with those of incompressible counterparts, whenever comparisons are made. Hence, we conclude that weak compressibility of $\langle\delta \rho / \rho\rangle_{\text {rms }}$ up to $\sim 0.45$ would not be important in characterizing MHD turbulence.

2. Converged behavior in simulated turbulence, such as that of the SF class, starts to appear only in simulations with very high resolution, $1024^{2}$ grid cells or more, in our simulations. The fact that simulations with $1024^{2}$ grid cells or more are necessary for our two-dimensional study of MHD turbulence points to the need for very high resolution in three-dimensional MHD turbulence studies as well. For hydrodynamic turbulence, Porter, Pouquet, \& Woodward (1998) reported that converged results emerged with $512^{3}$ or more grid cells in their simulations. However, we note that numerical dissipation would differ in different codes based on different schemes. As a result, the convergence behavior would be different, too. These findings emphasize the importance in turbulence studies of high-resolution grids and codes with the smallest possible numerical dissipation. Any explicit treatment of dissipation will require even higher resolution simulations, since numerical dissipation and diffusion would have to be smaller than the explicit terms.

The work by H. L. and D. R. was supported by an international collaboration grant from Korea (KOSEF F01-2000-000-10009-0) and Germany (Deutsche Forschungsgemeinschaft). The work by J. K. was supported by the Strategic National Research and Development Program (M10222000005-02B0600-00400) from the Ministry of Science and Technology, Korea. The work by T. W. J. was supported by the National Science Foundation (AST 96-19438; AST 00-71167) and the University of Minnesota Supercomputing Institute. Simulations were made through the support of The Supercomputing Application Support Program of KISTI.
Beck, R., Brandenburg, A., Moss, D., Shukurov, A., \& Sokoloff, D. 1996, ARA\&A, 34, 155

Biskamp, D. 1993, Nonlinear Magnetohydrodynamics (Cambridge: Cambridge Univ. Press)

Carilli, C. L., \& Taylor, G. B. 2002, ARA\&A, 40, 319

Cattaneo, F. 1994, ApJ, 434, 200

Cattaneo, F., \& Vainshtein, S. I. 1991, ApJ, 376, L2

Clarke, T. E., Kronberg, P. P., \& Böhringer, H. 2001, ApJ, 547, L111

Kim, J., Ryu, D., Jones, T. W., \& Hong, S. 1999, ApJ, 514, 506

Kronberg, P. P. 1994, Rep. Prog. Phys., 57, 325

Kulsrud, R. M. 1999, ARA\&A, 37, 37

Kulsrud, R. M., Cen, R., Ostriker, J. P. \& Ryu, D. 1997, ApJ, 480, 481

Lesieur, M. 1997, Turbulence in Fluids (3d ed.; Dordrecht: Kluwer)

Porter, D. H., Woodward, P. R., \& Pouquet, A. 1998, Phys. Fluids, 10, 237
REFERENCES

Ruzmaikin, A. A., Shukurov, A. M., \& Sokolov, D. D. 1988, in Magnetic Fields of Galaxies (Astrophys. \& Space Sci. Library 133; Dordrecht: Kluwer)

Ryu, D., Kang, H., \& Biermann, P. L. 1998, A\&A, 335, 19

Spitzer, L. 1979, Physical Processes in the Interstellar Medium (New York: Wiley)

Tao, L. Cattaneo, F., \& Vainshtein, S. I. 1993, in Theory of Solar and Planetary Dynamos, ed. M. R. E. Proctor, P. C. Matthews, \& A. M. Rucklidge (Cambridge: Cambridge Univ. Press), 303

Zweibel, E. G., \& Heiles, C. 1997, Nature, 385, 131

Zweibel, E. G., Heitsch, F., \& Fan, Y. 2002, in Turbulence and Magnetic Fields in Astrophysics, ed. E. Falgarone \& T. Passot (Berlin: Springer), 101 